\title{
TWAN: A way of networking third-world astronomers
}

\author{
Jayant V. Narlikar \\ Inter-University Centre for Astronomy and Astrophysics, Ganeshkhind, Pune 411007, India \\ email: jayant@iucaa.ernet.in
}

\begin{abstract}
This talk describes a proposal to set up a series of international institutions in different parts of the world to serve as nodes in a network that links astronomers from the developing nations worldwide. This network, along with its nodes is visualized as an economic way of upgrading the facilities for teaching, research and development of astronomy in the Third World countries. By way of illustration, the modus-operandi of the Inter-University Centre for Astronomy and Astrophysics in Pune, India is described. A network of this kind is suggested as a cost-efficient way of sharing limited resources.
\end{abstract}

Keywords. Third World Astronomy Network (TWAN); IUCAA, India

\section{Introduction}

The idea of a Third World Astronomy Network (TWAN) was aired first at the special session of the IAU General Assembly held in Manchester in 2000 (see Narlikar 2001a). I will first briefly review the basic idea as proposed then.

The aim of TWAN is to bring together astronomers from third world countries together in a network that is empowered to provide them with assistance to improve their research, teaching and developmental facilities. The network concept helps share limited resources in a cost-effective way, using the benefits of the emerging communications technology.

The basic structure of TWAN can be visualized as a group of nations in a region which are linked together and serviced by a 'node', the nodes themselves being connected to one another in a worldwide network. A typical node is an international centre or institute that carries out certain mandated activities to serve the above-mentioned aim of TWAN.

By way of illustration, I mention two institutions that carry out activities somewhat similar to what is intended for the TWAN nodes. These are the Abdus Salam International Centre for Theoretical Physics (AS-ICTP) in Trieste, Italy and the Inter-University Centre for Astronomy and Astrophysics (IUCAA) in Pune, India.

The AS-ICTP was set up more than four decades ago by Abdus Salam, a distinguished theoretical physicist who hailed from Pakistan. From his own personal experience Salam had felt the need to create an international resource centre which would help physicists from the Third World nations to fulfill their aspirations of carrying out top class research. To this end the ICTP was created in Trieste in 1964 and it has served as a place where Third world scientists can visit to use the Centre's library and other resources, attend schools and workshops and meet scientists from other countries to forge new collaborations. Since its inception, the ICTP has expanded its sphere of subjects beyond theoretical physics. The centre is supported largely by the Italian Government as well as by UNESCO and the International Atomic Energy Agency. It was named after its founder Professor Salam, after he passed away in 1996. For details of how the centre came about, see the interesting article by Professor Salam (1990). 
The IUCAA was set up in 1988 as a centre of excellence by the University Grants Commission of the Government of India, with the mandate that the centre shall provide facilities and guidance to astronomers and astrophysicists from Indian universities in their research, teaching and developmental activities. Since the IUCAA was specifically aimed at astronomers and astrophysicists, it comes close to what is being proposed for a TWAN node. I will therefore spend some time on describing how the IUCAA functions.

\section{What does IUCAA do?}

For those interested in the details of what led to the setting of IUCAA and the impact it has had on university education and research, I may refer to my article published a few years ago (Narlikar 2001b). Briefly, I may add that the creation of IUCAA was motivated by the growing gap in the standards of academic functioning of universities on the one hand and autonomous research institutes (ARIs) on the other. The latter had been created largely since Indian independence in 1947, were generally limited to their specific subject areas and had scientists carrying out research and developmental activities unburdened by teaching graduates and undergraduates. Compared to universities the ARIs were much better endowed, had better facilities and better working conditions.

Although the universities also proliferated in number, their quality steadily went down. The academics at the universities were required to spend most of their time on lecturing, leaving very little scope for research and development. The funds for the latter also shrank so that the balance of research began to tilt heavily towards the ARIs.

To redress this balance the University Grants Commission, the parent funding agency for universities in India, came up with the idea of setting up inter-university centres (IUCs). In a typical IUC a subject that is not very well funded in a typical university is taken up. For example, the IUCAA was created to support astronomy and astrophysics (A\&A). Given that there are 200+ universities, it would be prohibitively expensive to provide all A\&A-related facilities to each and every university. Moreover, if the number of research workers (teachers and students included) in a typical university were no more than 20-30, these facilities would remain largely under-utilized. So a way had to be found whereby precious resources would be maximally used. The IUC mode is such a way: it is endowed liberally with the necessary resources and it ensures that they are optimally used by the university academics. How is this done?

Let me briefly describe the in-house facilities at IUCAA. It contains an up to date A\&A library which also contains books of peripheral interest to astronomers, like physics, mathematics, computer science, etc. It has a good stock of A\&A and physics journals and electronic subscription to many. It has mirror images of leading data centres and is currently playing a lead role in the Indian contributions to the international Virtual Observatory programme. Then there is a computer centre with state of the art software for use by astronomers as well as by theoreticians. To help development of instruments, there is a laboratory that guides users in making various astronomical instruments. Recently, a new-technology telescope with $2 \mathrm{~m}$ aperture has been set up not far from Pune which will provide observing experience to astronomers from universities.

I should also mention the circumstance that influenced the siting of IUCAA in Pune. Its location is next door to the National Centre for Radio Astrophysics (NCRA) of the Tata Institute of Fundamental Research. The NCRA has been operating the Giant Metrewave Radio Telescope (GMRT) situated around $90 \mathrm{~km}$ from Pune. The nearness to this national centre also provides IUCAA and its university visitors with additional facilities and guidance.

How do university astronomers use these facilities? To facilitate this, IUCAA has introduced an associateship programme along the lines of AS-ICTP. This programme identifies 
active and promising university scientists as prospective users of IUCAA by making them visiting associates. A visiting associate can visit IUCAA frequently and for extended periods for using its facilities for his/her research. A senior research worker from a university who is a visiting associate can also bring his student with him if he feels that the student would benefit by such a visit. All costs for such visits are borne by IUCAA.

Following the AS-ICTP, the IUCAA has also an annual calendar of pedagogical activities. It runs a graduate school jointly with NCRA, to which university students are also invited. There are introductory schools or workshops in A\&A as well as advanced research level meetings. Resource persons for such meetings are drawn from all over India and also sometimes from abroad.

Given a year-round influx of visiting scientists and students at IUCAA, an important facility of course is the hostel and guest house. These are attached to the main IUCAA buildings so that the visitors need spend very little time commuting to work. Long term visitors are accommodated in guest apartments. Having such in-house facility greatly helps the efficiency of the visiting scientist.

Another aspect of IUCAA deals with encouraging astronomical observing at the professional level. University scientists have been encouraged to go out of their campuses to carry out observations that relate to their research. Not only national facilities like the GMRT, the Vainu Bappu Telescope in the south or the ARIES Observatory in the north: the IUCAA encourages going beyond the national boundary to participate in guest observing programmes on major telescopes abroad. This is done through collaborations forged with scientists abroad. IUCAA helps and advises the university scientists in practical matters such as writing a proposal for observing.

To be able to carry out these tasks, IUCAA has provision for a resident faculty of active research workers who also carry out pedagogical activities. In order that IUCAA's guidance commands credibility, the faculty members have to be seen to belong to the top echelons in their respective subject areas. This faculty also has research students to guide and is supported by a vigorous post-doctoral programme. Indeed, from early days IUCAA's post-doctoral programme has had an international flavour with post-docs from Japan, China, France, Italy, the USA, the UK, Iran, etc.

Last, but not the least, IUCAA runs various programmes of public outreach, such as public lectures, night sky viewing, special viewing programmes at eclipses or transits, workshops for making telescopes for amateur observing, etc. Knowing the importance of attracting and motivating students towards science and astronomy in particular, IUCAA has an activity centre for school students around which many programmes are built. On the second and fourth Saturdays for example, there are morning lectures for students from secondary and higher secondary levels. Well illustrated and with demonstrations to accompany them, these lectures are very popular and the 500-seater auditorium is often overflowing for such events.

During the summer vacations IUCAA invites interested school students to do projects with the IUCAA staff. In a week long period a student (or a group of students) complete a project which teaches them some science. A special occasion is the National Science Day, when IUCAA stages an open house for the general public, which turns up in thousands. On this day we also have science quiz and competitions for school children.

\section{The impact of IUCAA}

What has been the impact of IUCAA? Several indicators suggest that the experiment has been remarkably successful. One ready reckoner is the list of publications of university academics. The number of papers published in standard refereed journals have multiplied 


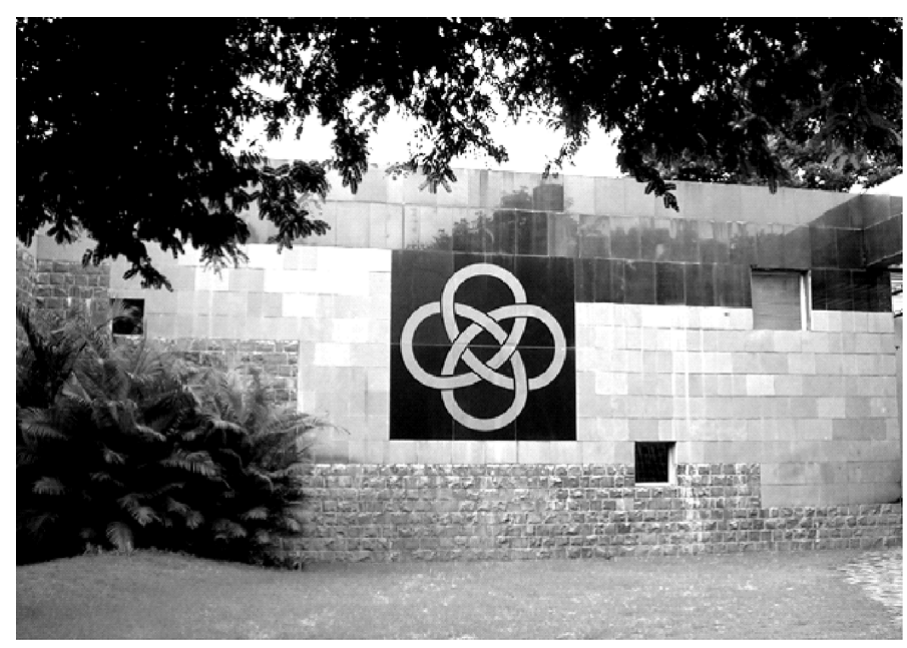

Figure 1. IUCAA's symbol displayed on the wall at the main entrance emphasizes its networking with the universities. It also suggests that like the loop without ends, astronomers are engaged in an unending cosmic search.

several times. The number of university departments offering A\&A at post graduate level has also trebled since before IUCAA started.

The guest-observing programmes have brought international level observing to many university campuses. One of the speakers at this meeting (S.K. Pandey) started off as a theoretical solar physicist in a small university; but after collaborations with the IUCAA faculty, has blossomed into an observational astronomer with experience of observing galaxies, using many telescopes abroad.

The associateship programme brings faculty members from many different universities to the IUCAA campus at the same time. This circumstance, arising because there are common vacation windows for many universities, has led to collaborations between scientists who otherwise would not have met.

Instrumentation had long remained a neglected area in universities and most university scientists were forced by the lack of experimental facilities to be theorists. With the guidance provided by IUCAA's instrumentation laboratory, the culture of instrumentation has been growing.

IUCAA's public outreach programme has made the institution well known in the city of Pune and the state of Maharashtra. Its school-student oriented programmes have created a strong base of young amateur astronomers in the state and has inspired students to perform very well in the astronomy Olympiads.

Perhaps, it is necessary to recognize that IUCAA was the first Indian scientific institution to have instituted a scientific advisory committee with an international flavour, to oversee its programmes and monitor its performance. The SAC-IUCAA has provided valuable guidance that has helped IUCAA progress towards its objectives in more efficient ways.

\section{A TWAN node}

I have gone into details in describing IUCAA, because I feel the model of this institution comes very close to what we expect from a node of TWAN, and can be easily adapted to its requirements. Read 'third world countries' for 'universities' in the above scenario, and expand the 'catchment area' of the node to cover several countries in the region. Thus a 
node in India can very well serve the Asia-Pacific region, which, of course may require an additional node because of its geographical spread.

A typical node should have at least 6-10 experienced and productive astronomers on its staff carrying front-line research, who would guide the node's academic programmes. To support them, the node would be expected to have the following in-house academic facilities:

\section{A very good library}

2. An advanced computer centre

3. A data-mining facility

4. An R\&D centre for instrumentation

5. Access to a good observing facility

6. A guest house for visitors

What will be the academic programmes that a node should be mandated to offer? Going by the example of IUCAA, the following suggest themselves:

1. Associateship programme: An associateship programme along the lines of IUCAA or AS-ICTP should be introduced. This should provide for, say, one visit to the node in two years over a period of 6 years for the associate. The duration of a visit should be at least for one month and may extend up to six months at a time. Associates may be selected from the region under the node and the node will take care of travel and subsistence of the associate for this visit.

2. Visits by third world academics: A scheme whereby a 1-3 month visit to the node by an active senior academic from the region may be encouraged. Unlike the associateship programme, this would be a one-off visit, with its academic objective well defined beforehand. The node may have an arrangement with the Third World Academy of Sciences which supports visits of this kind.

3. Schools for young astronomers: The node may take up the responsibility of organizing international schools for young astronomers coming from the region. This will be similar to the ISYA of the IAU, but limited to the region under the node.

4. Advanced workshops: To encourage research workers in a specific area of A\&A, advanced schools may be arranged by the node. A workshop can be of 3-4 week duration and the lecture notes based on it may be published/or widely circulated through node's website.

5. Guest observer programme: The faculty of the node should actively encourage participation of the region's astronomers in guest observing in international observing facilities. The faculty may also advise the user from the region on data-mining and usage of virtual observatory facilities.

6. Instrumentation programme: The node should provide guidance in making instruments for astronomical observing. The role of the node staff should be catalytic in the sense that the actual instrument building would be done by the astronomers of the region.

The node should be governed by an Executive Council having representatives of the funding agencies, some user nations of the region and a few experienced astronomers. It should also have a scientific advisory committee of distinguished astronomers who are sympathetic to the objectives of TWAN. An ideal funding arrangement suggested here (along the lines of the AS-ICTP) is through the International Astronomical Union, UNESCO and the country in which the node is sited. 


\section{Budgetary aspects}

How much will such a centre cost to set up? How much will be its running cost? These matters will need to be looked at in detail. What are presented here are tentative figures based on my IUCAA experience. If such a centre were set up in India, I expect that the cost of buildings and the in-house facilities will come up to around US\$ 10 million. It is assumed that the Government of India would provide land at no cost or at a nominal lease rent (as is often done). The Government may also be requested to bear the cost of the building. So far as the running cost is concerned, at rates prevalent in India, we arrive at the following figures for annual running costs in US\$:

10 Faculty Members

250000

20 Infrastructural staff (10 employees +10 on contract) 250000

$\begin{array}{ll}\text { Maintenance, utilities, etc. } & 100000\end{array}$

Academic facilities (as described in section 4) 200000

Academic programmes (as described in section 4) 200000

Total annual budget

1000000

If IUCAA were entrusted to carry out the functions of a node until an independent one were set up, the annual cost may work out close to US\$200000. This is because IUCAA already has most of the infrastructure in place and will need a supplementary grant to take on the additional responsibility of a TWAN node.

\section{Concluding remarks}

The worldwide network envisaged under the auspices of TWAN will require more such nodes to take care of other regions. I expect there may be as many as 6 nodes to cover the developing countries in Asia, Africa and Latin America. The costs, compared to what is needed to create a major observing facility are modest, but the dividend in terms of upgrading the output of third world astronomers will be very significant.

I hope that this idea is taken up by the International Astronomical Union as a worthwhile activity to support. An ideal time for launching a TWAN node is the year 2009, the proposed International Year for Astronomy.

\section{Acknowledgements}

I thank the IAU for giving me the opportunity for airing my vision of TWAN. At a personal level I thank Alan Batten and John Hearnshaw for their backing to the project.

\section{References}

Salam, A. 1990, in Proceedings of the 25th anniversary conference 'Frontiers in Physics, High Technology and Mathematics', eds. Cerdeira, H.A. and Lundqvist, S.O. (World Scientific), 74-81

Narlikar, J.V. 2001a, in Proceedings of the IAU Special Session, ed. Alan H. Batten (Astronomical Society of the Pacific), 324-328

Narlikar, J.V. 2001b, in Organizations and Strategies in Astronomy, Vol 2, ed. Andre Heck (Kluwer, Dordrecht), 29-45 